\title{
MOR of Piezoelectric Beam FEM Model and its Control
}

\author{
Vladimír Kutiš̀ ${ }^{\dagger}$ Juraj Paulech ${ }^{\dagger}$, Gabriel Gálik ${ }^{\dagger}$, Justín Murín ${ }^{\dagger}$ and Vladimír Goga ${ }^{\dagger}$ \\ † Department of Applied Mechanics and Mechatronics \\ Institute of Automotive Mechatronics \\ Faculty of Electrical Engineering and Information Technology \\ Slovak University of Technology \\ Ilkovicova 3, 81219 Bratislava, Slovak Republic \\ Email: vladimir.kutis@stuba.sk - Web page: http://www.uamt.fei.stuba.sk
}

\begin{abstract}
Smart materials are a very important building blocks in mechatronic applications. These materials include shape memory materials, magnetorheological, functionally graded, piezoelectric, and other types of modern materials. Their main feature is that they make it possible to extend the functional use of a given system, modify the dynamic characteristics of a given system or allow a return to the original state of the system under certain conditions.

Frequently used smart materials are piezoelectric materials, which couple the mechanical and electrostatic fields. This physical coupling predestines piezoelectric materials for use as sensors or actuators [1], as they allow the mechanical deformation to be converted to an electric potential and electrical potential for mechanical deformation, respectively. Such use of piezoelectric materials in mechatronic systems is closely connected to control systems and controller design according to defined requirements. When controlling mechatronic systems, the so-called modern control, i.e., state space control is considered [2]. State space models of given physical systems are used in the design of state space controllers. From a control point of view, it is very desirable that the state model is not too large. The finite element method is most often used for the physical description of a given system, but the created FEM model is dimensionally significantly larger than the state model suitable for control purposes. It is therefore necessary to reduce the FEM model, i.e., use one of the reduction methods and then transform the reduced FEM model into a state space model.

This paper deals with the development of the FEM model of piezoelectric beam elements, where the piezoelectric layers are located on the outer surface of the beam core, which is made of functionally graded material. Subsequently, the FEM model created in this way is reduced by the so-called exact method, which is one of the MOR methods. Next MOR FEM equations are transformed into a state space model. Created state space model is connected to LQR controller and the reduced system is analysed. Developed FEM model, MOR technique, state space model and LQR control are implemented in FEM code MultiFEM.
\end{abstract}

\section{ACKNOWLEDGEMENT}

This work was supported by the Slovak Grant Agency: VEGA No. 1/0081/18, KEGA 011STU-4/2020 and APVV-19-0406.

\section{REFERENCES}

[1] S. Moheimani and K. A. Fleming, Piezoelectric Transducers for Vibration Control and Damping, Springer, (2006).

[2] B. Friedland, Control System Design, An Introduction to State-Space methods, Dover, (1986). 